\title{
Caracterización demográfica de la población con esófago de Barrett en dos instituciones médicas de Bogotá, Colombia
}

\section{Demographic characterization of the population with Barrett's esophagus in two medical centers of Bogotá, Colombia}

\author{
María Lucía Bernal-Vaca, MD,,$^{*}$ (10) Harol Felipe García, MD, ${ }^{2}$ (D) Belén Mendoza de Molano, MD. ${ }^{3}$ (D)
}

\author{
GacCeso abierto \\ Citación: \\ Bernal Vaca ML, García HF, Mendoza de Molano \\ B. Caracterización demográfica de la población \\ con esófago de Barrett en dos instituciones \\ médicas de Bogotá, Colombia. Rev Colomb \\ Gastroenterol. 2020;35(3):311-318. https://doi. \\ org/10.22516/25007440.488
}

Médica egresada de la Universidad de los Andes. Residente de Medicina Interna, Universidad del Rosario. Durante la ejecución del estudio: interna, Universidad de los Andes. Bogotá D. C., Colombia.

Médico egresado de la Universidad de los Andes. Durante ejecución del estudio: interno, Universidad de los Andes. Bogotá D. C., Colombia.

${ }_{3}^{3}$ Medicina Interna, Gastroenterología, Endoscopia digestiva y Fisiología digestiva. Sección de Gastroenterologia, Endoscopia, Hepatología y Fisiología en Hospital Universitario Fundación Santa Fe de Bogotá Bogotá D. C., Colombia.

"Correspondencia: María Lucía Bernal-Vaca, MD ml.bernal11@uniandes.edu.co

Fecha recibido: $\quad 06 / 12 / 19$ Fecha aceptado: 16/06/20

\begin{abstract}
Resumen
Introducción: el esófago de Barrett es un trastorno en el que ocurre un cambio del epitelio escamoso estratificado del esófago por uno columnar especializado, lo cual se da como consecuencia del reflujo gastroesofágico crónico. En Colombia no se conoce la prevalencia actual de esta patología, ni se ha caracterizado a la población que la padece. El presente estudio tiene como objetivo conocer cuáles son las características demográficas principales de la población diagnosticada con esófago de Barrett en dos instituciones médicas de Bogotá. Material y métodos: se realizó un estudio de corte transversal multicéntrico, en el cual se evaluaron los reportes de endoscopias y de histopatología de 3000 pacientes que asistieron a estas instituciones por cualquier indicación. A partir de estos reportes se tomaron los datos requeridos. Asimismo, se realizó un análisis estadístico descriptivo de dichos datos. Resultados: la prevalencia del esófago de Barrett en la muestra es del 0,73\%. Se observó, además, que la correlación endoscópico-patológica es baja (28,5\%). De los casos diagnosticados, el rango de edad más frecuente se ubica entre los 60 y 80 años, con una edad promedio de 65,5 años. Asimismo, existe una predominancia de esta patología en el sexo femenino (63,6 \%), en personas con un índice de masa corporal (IMC) $>25 \mathrm{~kg} / \mathrm{m}^{2}$ y en aquellas con antecedentes de tabaquismo, sin historial de consumo de alcohol. En la mayoría de pacientes, se realizó la endoscopia por síntomas de reflujo gastroesofágico $(50 \%)$. La longitud del segmento observado no fue reportada en una gran cantidad de endoscopias. Conclusiones: en las instituciones analizadas, el esófago de Barrett es una patología de muy baja prevalencia y predominante en mujeres de edad avanzada con síntomas de reflujo gastroesofágico, sobrepeso y antecedente de tabaquismo.
\end{abstract}

Palabras clave

Esófago de Barrett, sexo, distribución por edad, signos y síntomas, prevalencia.

\section{Abstract}

Introduction: Barrett's esophagus occurs when the stratified squamous epithelium of the esophagus changes to a specialized columnar epithelium as a result of chronic gastroesophageal reflux. Its current prevalence in Colombia is unknown and the population suffering from it has not been characterized. The present study aims to determine the main demographic characteristics of the population diagnosed with Barrett's esophagus treated at two medical centers in Bogotá, Colombia. Materials and methods: A multicenter cross-sectional study was conducted to assess the endoscopy and histopathology reports of 3,000 patients who underwent this procedure for any reason. A descriptive statistical analysis of the data was performed. Results: The prevalence of Barrett's esophagus in the sample was $0.73 \%$. The endoscopic-histology correlation was low $(28.5 \%)$. Of the diagnosed cases, the most frequent age range was $60-80$ years, with an average age of 65.5 years. This condition is predominant in the female sex (63.6\%), in people with a BMl over $25 \mathrm{~kg} / \mathrm{m}^{2}$, with a history of smoking, and no history of alcohol consumption. Most patients underwent endoscopy for symptoms associated with gastroesophageal reflux (50\%). The length of the observed segment was not reported in most endoscopies. Conclusions: In the medical centers included in this study, Barrett's esophagus is a rare pathology, found predominantly in elderly women with symptoms of gastroesophageal reflux, overweight, and with a history of smoking.

\section{Keywords}

Barrett's esophagus: Sex; Age distribution; Signs and symptoms; Prevalence. 


\section{INTRODUCCIÓN}

El esófago de Barrett (EB) es el factor etiológico más importante para el desarrollo de un adenocarcinoma de esófago (1). Es importante diagnosticar el EB, dado que en estos pacientes el riesgo de adenocarcinoma de esófago aumenta entre 10 y 30 veces con respecto a la población general (2-4). En este contexto, la posibilidad de que se forme un adenocarcinoma en los pacientes con EB es mayor en el sexo masculino y en personas de edad avanzada, así como en enfermos con una duración del EB >10 años, con presencia de esofagitis y con EB de segmento largo (5-7).

En un estudio realizado en la Fundación Santa Fe de Bogotá, la incidencia de cáncer esofágico, después de 11 años de seguimiento a pacientes con $\mathrm{EB}$, fue del $4 \%$ (8). Recientemente, se ha estimado una incidencia anual de adenocarcinoma de esófago en pacientes con EB que oscila entre el 0,2 y el $0,3 \%(9,10)$.

Dentro de ese panorama, el EB no es una patología poco común y, de hecho, se encuentra en el $2 \%$ de la población general de otros países (11), así como en el 10-15\% de pacientes con reflujo gastroesofágico $(1,12)$. Incluso, en individuos asintomáticos mayores de 50 años, la prevalencia puede alcanzar el $25 \%$ (13). En vista de estas cifras considerables, es importante conocer cuál es la prevalencia de esta enfermedad en Colombia.

En efecto, en Colombia no se conoce la prevalencia actual del EB, según la literatura revisada, ni tampoco se ha caracterizado a la población que la padece, razón por la cual es importante investigar al respecto. Al considerar que los datos de epidemiología en nuestro país son limitados, y que esta es una patología precursora del adenocarcinoma de esófago, el cual puede tener una supervivencia del $17 \%$ a 5 años (14), consideramos fundamental conocer las características de los pacientes que cursan con la enfermedad y conocer realmente cuál es su prevalencia en el territorio nacional.

Con todo este contexto, ampliar el conocimiento epidemiológico de la patología nos permitiría optimizar el diagnóstico mediante la caracterización de la población, así como conocer, a partir de las cifras de prevalencia, la importancia de esta enfermedad en Colombia.

Por tanto, el presente estudio apunta a conocer cuáles son las características demográficas principales de la población diagnosticada con EB en dos instituciones médicas de Bogotá. En cuanto a los objetivos secundarios, buscamos determinar la prevalencia del EB en la población objeto, además de determinar la proporción entre el EB de segmento corto y largo en los casos confirmados y analizar la correlación endoscópico-patológica.
MATERIAL Y MÉTODO

\section{Tipo de estudio}

Se realizó un estudio de corte transversal, con objetivos anotados previamente.

\section{Población objeto}

Pacientes que asistieron a las dos instituciones para realizarse una endoscopia de vías digestivas altas, entre marzo y mayo de 2018. Se escogieron estas dos instituciones con el fin de incluir en la población objeto un espectro más amplio de prestadores de servicio y condiciones socioeconómicas. Asimismo, se incluyeron los pacientes que se sometieron a una endoscopia de vías digestivas altas y se excluyeron aquellos que tuvieran diagnóstico de adenocarcinoma de esófago o antecedente de tratamiento del EB con displasia. Entonces, tomamos 3000 pacientes que cumplieran con dichos criterios. Este tamaño de muestra fue calculado a partir de un intervalo de confianza (IC) del $95 \%$, una prevalencia estimada del $2 \%$ y una precisión del 0,5\%. En efecto, la prevalencia estimada se tomó de un estudio realizado en México (11), en el cual la prevalencia del EB en la población general sometida a endoscopia fue del 1,8 \%.

\section{Fuente de la información}

Se revisaron los reportes de endoscopia, de histopatología y las historias clínicas de los pacientes seleccionados, a partir de los cuales se tomaron los datos necesarios. Específicamente a partir de los reportes de patología, registramos en qué porcentaje de pacientes con hallazgos endoscópicos se confirmó metaplasia intestinal en la biopsia. Se computó cuántos tuvieron diagnóstico definitivo del EB y cuántos no, lo cual utilizamos para el cálculo de prevalencia del EB en la población estudiada.

\section{Análisis de la información}

Para el procesamiento de datos, utilizamos Excel versión 2017. Se realizó un análisis descriptivo de información a través de media, mediana, moda y desviaciones estándar. Pudimos observar la distribución del desenlace según las características seleccionadas.

Además, se determinó la prevalencia endoscópica de la patología en cuestión, tomando como población total los 3000 pacientes seleccionados y ubicando en el numerador el número de personas en quienes se realizó el diagnóstico de EB. 
Número de pacientes con diagnóstico de EB

(endoscopia + biopsia)

3000 pacientes en quienes se realizó una

endoscopia de vías digestivas altas (EVDA)

\section{RESULTADOS}

Durante el período de estudio, 3000 pacientes fueron sometidos a endoscopia de vías digestivas altas, entre marzo y mayo de 2018: 1500 se remitieron a una institución y los 1500 restantes se condujeron a la otra. Las endoscopias fueron realizadas, en el total de los casos, con el videoendoscopio Olympus EVIS Exera III-CLV 190, el cual proporciona una imagen de alta definición.

De los pacientes, 1633 (54,4\%) eran mujeres y 1367 $(45,6 \%)$, hombres, con una edad promedio de 51 años (mínimo 15 y máximo 93 años). Las indicaciones más frecuentes para el examen endoscópico fueron, en primer lugar, los síntomas de reflujo gastroesofágico, que correspondieron al 16,9 \% de los participantes (508 casos); también se incluyeron la epigastralgia, con el 10,8 \% (324 casos), y antecedente endoscópico de gastritis, 6,7 \% (201 casos). No se mencionó la indicación de la endoscopia en el 13,5\% (405) de los reportes.

Sobre la base de los hallazgos endoscópicos, se sospechó la existencia de EB en 77 pacientes $(2,5 \%$ de la muestra total). De ellos, en 22 se confirmó una metaplasia intestinal tras la histopatología. Esto quiere decir que la correlación endoscópico-patológica fue del $28,5 \%$, y se descartó el EB en 52 pacientes $(67,5 \%)$. Asimismo, $2(2,5 \%)$ reportes de patología no fueron encontrados, y en 1 paciente $(1,2 \%)$ no pudo tomarse la biopsia debido a un sangrado digestivo activo (Figura 1).

Entre tanto, en un $70 \%$ de los pacientes con sospecha de EB se tomaron 1 o 2 biopsias, mientras que en el $30 \%$ se realizaron de 3 a 6 biopsias. En el 84,7 \%, la biopsia fue tomada de la línea $Z$, y en el $10,86 \%$ no se mencionó el lugar preciso de toma de la biopsia en el reporte. El 93 \% de las histopatologías fueron revisadas por un patólogo (Tabla 1).

En cuanto a la caracterización demográfica, de los 22 pacientes con diagnóstico confirmado, $14(63,6 \%)$ eran mujeres y $8(36,3 \%)$ eran hombres (Figura 2). El rango de edad de los pacientes con EB oscila entre los 40 y los 79 años, con una media de edad general de 65,5 años. El 77,2 $\%$ (17 pacientes) se encontraron dentro del rango de edad de los 60 a los 79 años, el 13,6\% (3) entre los 50 y 59 años y el $9 \%$ (2) entre los 40 y 49 años (Figura 3). Además, el $67 \%$ de los participantes tenían un IMC correspondiente a sobrepeso u obesidad, mientras que el $33 \%$ mostró un rango de peso normal (Tabla 2).

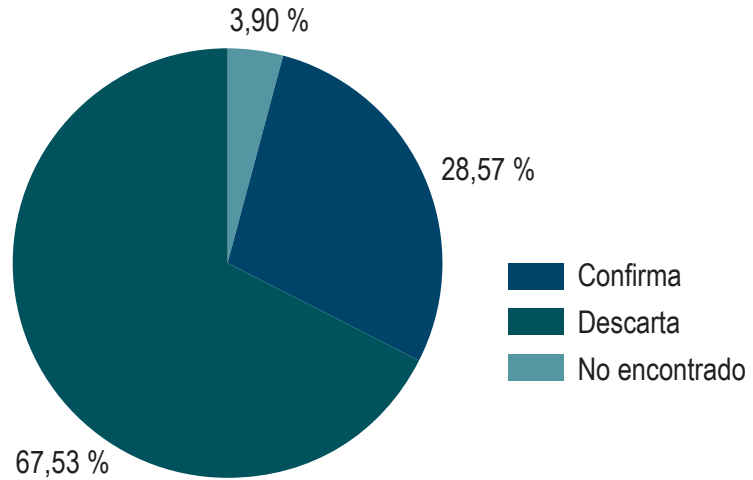

Figura 1. Correlación entre los hallazgos de la endoscopia de vías digestivas y el resultado de la patología. El $100 \%$ representa la totalidad de los pacientes en quienes se sospechó EB tras la endoscopia. Así, Confirma quiere decir que se confirmó el EB mediante patología; Descarta se refiere a que se descartó el diagnóstico de EB mediante patología, y no encontrada alude a que no se logró conocer el resultado de la biopsia o no se consiguió la realización de esta por algún motivo.

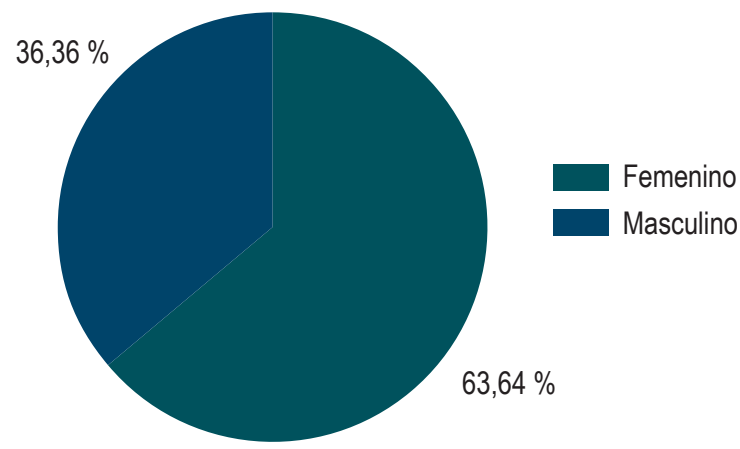

Figura 2. Sexo de los pacientes con EB. Del total, el 36,3 \% fueron hombres y el 63,6 \% fueron mujeres.

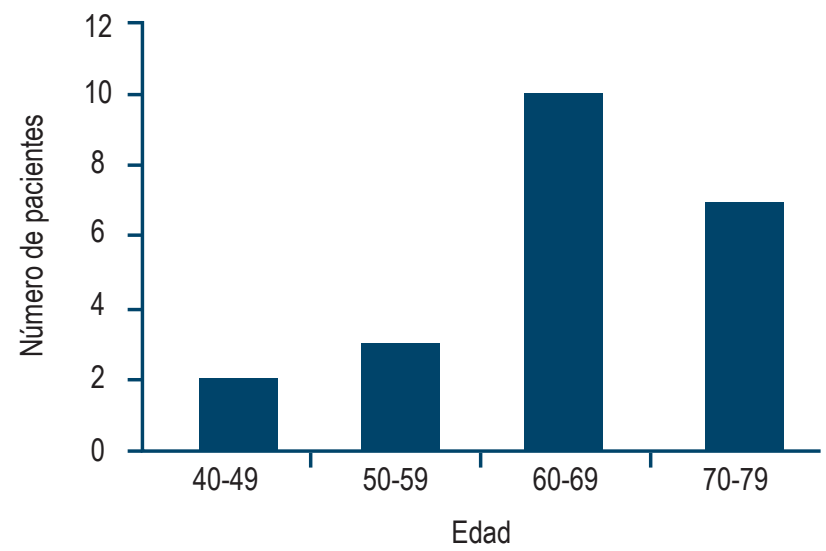

Figura 3. Edad de pacientes con EB. En el eje X se observan los rangos de edad: 40 a 49, 50 a 59, 60 a 69 y 70 a 79 años.

Con respecto a los antecedentes de consumo, el $50 \%$ de los pacientes presentaban tabaquismo (actual o suspen- 
Tabla 1. Estadísticas descriptivas con respecto a la endoscopia de vías digestivas y la histopatología de pacientes con sospecha o diagnóstico definitivo de EB

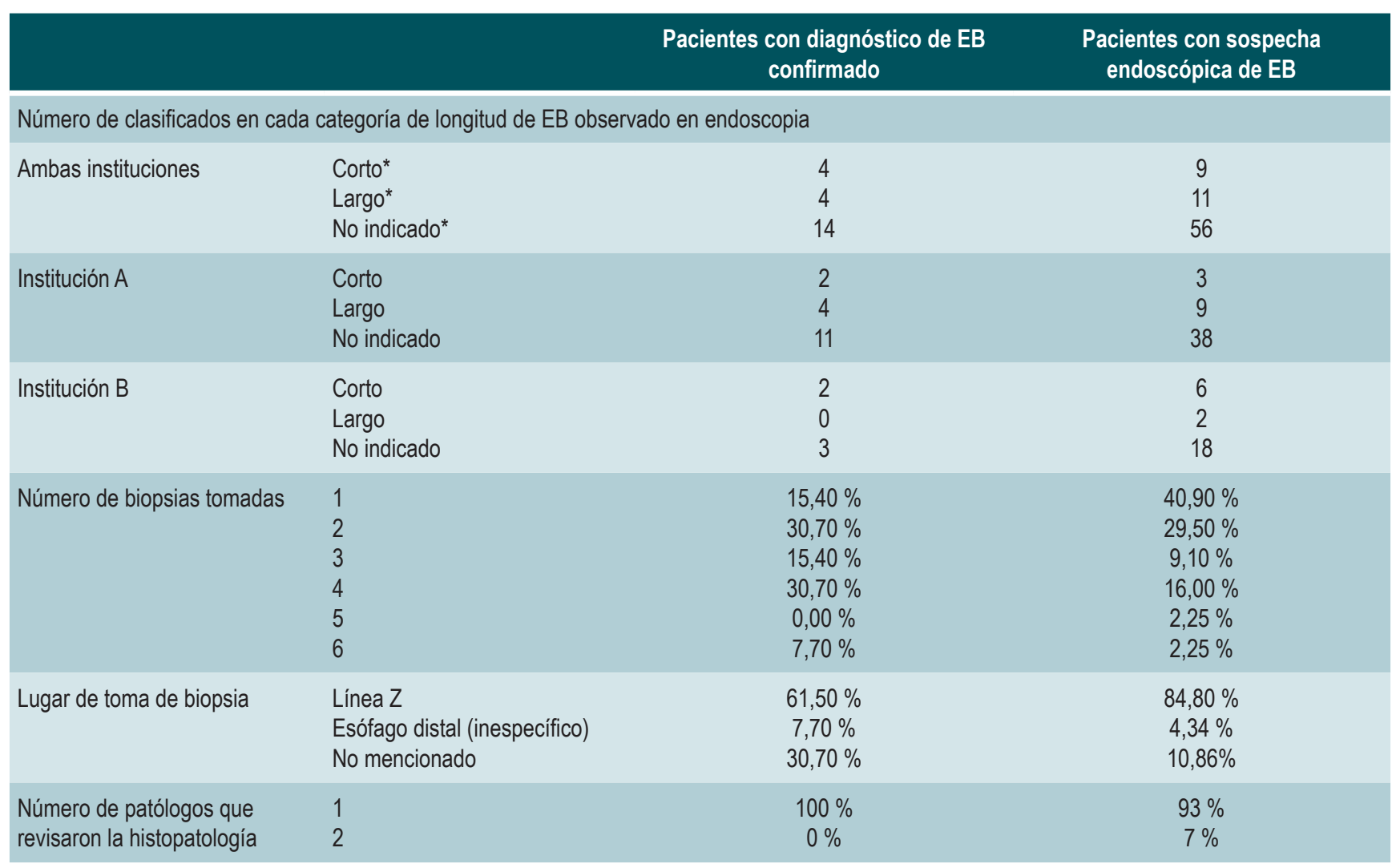

EB: esófago de Barrett. "Corto: $<3 \mathrm{~cm}$; largo: $>4 \mathrm{~cm}$; no indicado: no se mencionó en el reporte de endoscopia la longitud del segmento observado, sugestivo de EB.

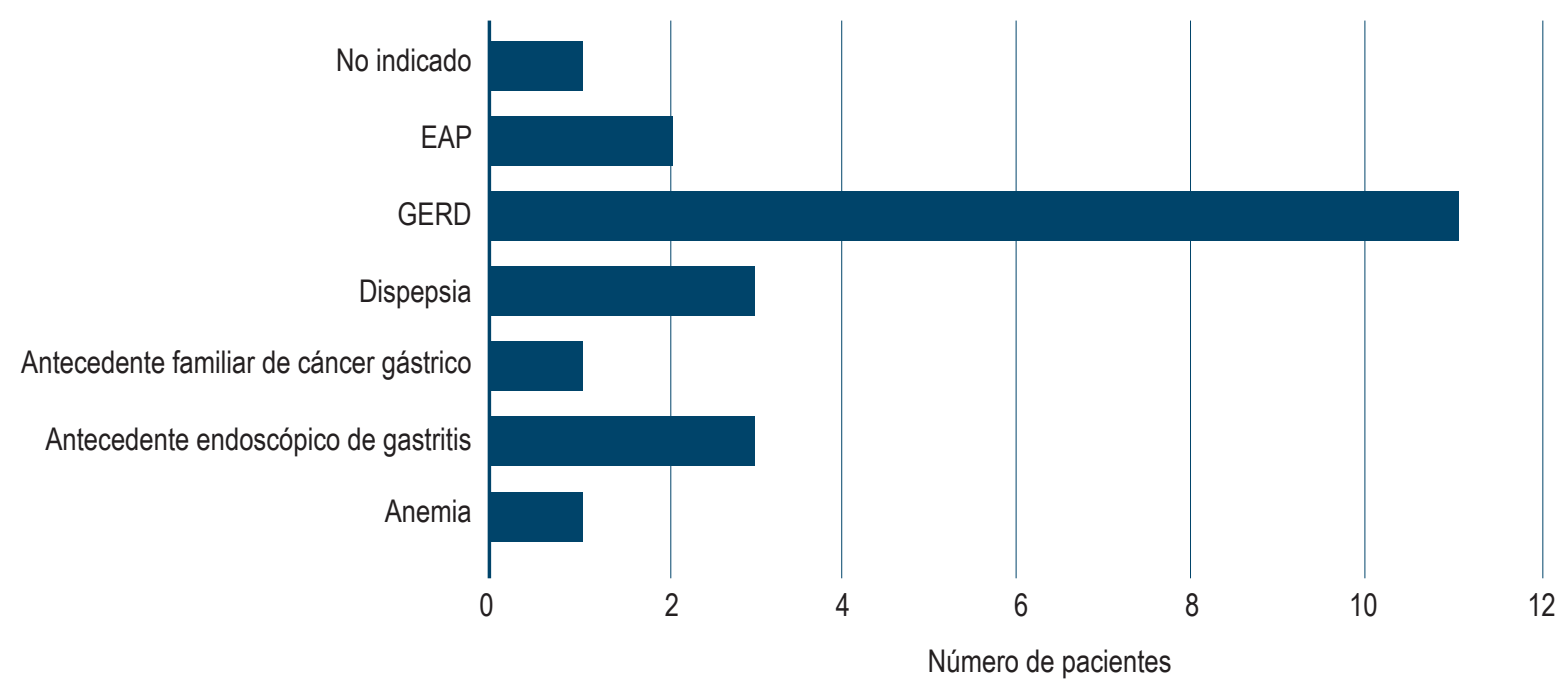

Figura 4. Indicación de endoscopia de vías digestivas en pacientes con EB. GERD: reflujo gastroesofágico crónico (Gastroesophageal Reflux Disease); EAP: enfermedad ácido péptica. No indicado: no se mencionó en el reporte de endoscopia el motivo por el cual se solicitó el estudio. 
dido), mientras que el $67 \%$ no tenían historia de consumo de alcohol. Sobre las condiciones sociodemográfica, el $58 \%$ correspondían a estratos 3 y 4 (Tabla 2).

De otro lado, la principal indicación de los pacientes con EB fue el reflujo gastroesofágico en 11 (50 \%) casos, dispepsia en $3(13,6 \%)$, antecedente endoscópico de gastritis en $3(13,6 \%)$, enfermedad ácido péptica en 2 (9\%), anemia en $1(4,5 \%)$, antecedente familiar de cáncer gástrico en $1(4,5 \%)$ y en $1(4,5 \%)$ no se había registrado ninguna indicación (Figura 4).

Tabla 2. Estadísticas descriptivas para diferentes variables personales de pacientes con EB

\begin{tabular}{|c|c|c|}
\hline \multicolumn{3}{|l|}{ Edad (años) } \\
\hline General & $\begin{array}{l}\text { Mediana } \\
\text { Media } \\
\text { Moda } \\
\text { Desviación }\end{array}$ & $\begin{array}{l}67,5 \\
65,59091 \\
59,69 \\
8,732311\end{array}$ \\
\hline Mujeres & $\begin{array}{l}\text { Mediana } \\
\text { Media } \\
\text { Moda } \\
\text { Desviación }\end{array}$ & $\begin{array}{l}67,5 \\
64,78571 \\
71,75 \\
10,31935\end{array}$ \\
\hline Hombres & $\begin{array}{l}\text { Mediana } \\
\text { Media } \\
\text { Moda } \\
\text { Desviación }\end{array}$ & $\begin{array}{l}67,5 \\
67 \\
69 \\
5,237229\end{array}$ \\
\hline $\begin{array}{l}\text { Índice de masa corporal } \\
\left(\mathrm{kg} / \mathrm{m}^{2}\right)\end{array}$ & $\begin{array}{l}<18 \\
18-24,9 \\
25-29,9 \\
30-34,99 \\
>35 \\
\text { Mediana } \\
\text { Media } \\
\text { Desviación }\end{array}$ & $\begin{array}{l}0 \% \\
33 \% \\
50 \% \\
17 \% \\
0 \% \\
24,66 \\
24,42 \\
3,027\end{array}$ \\
\hline Tabaquismo & $\begin{array}{l}\text { Exfumador* } \\
\text { Fumador actual* } \\
\text { No fumador* }\end{array}$ & $\begin{array}{l}41,60 \% \\
8,30 \% \\
50 \%\end{array}$ \\
\hline Consumo de alcohol & $\begin{array}{l}\mathrm{No}^{* *} \\
0-30 \mathrm{~g} / \mathrm{d}^{* *} \\
>30 \mathrm{~g} / \mathrm{d}^{* *}\end{array}$ & $\begin{array}{l}66,66 \% \\
33,33 \% \\
0 \%\end{array}$ \\
\hline Estrato socioeconómico & $\begin{array}{l}2 \\
3 \\
4 \\
5\end{array}$ & $\begin{array}{l}16,66 \% \\
25 \% \\
33,33 \% \\
16,66 \%\end{array}$ \\
\hline
\end{tabular}

"Exfumador: paciente con antecedente de tabaquismo, pero hábito actualmente abandonado; Fumador actual: hábito presente en el momento de la evaluación; no fumador: ausencia de antecedente de tabaquismo, ya sea en el pasado o en el presente

*No: consumo completamente ausente; $0-30 \mathrm{~g} / \mathrm{d}$ se modifica como 14 $\mathrm{g} / \mathrm{d}$ en mujeres; $>30 \mathrm{~g} / \mathrm{d}$ se modifica como $>14 \mathrm{~g} / \mathrm{d}$ en mujeres (valores tomados de la Organización Mundial de la Salud y el Instituto Nacional sobre Abuso de Alcohol y Alcoholismo)
Asimismo, el 8,1 \% de los pacientes presentaron EB de segmento corto, y el 18,1 \% tenían EB de segmento largo. Entre tanto, el 63,6 \% de los enfermos no tenían ningún tipo de categorización. Ninguno de los reportes de endoscopia revisados contenía el uso de alguna otra escala de clasificación. De igual forma, ninguna biopsia reveló displasia o malignidad en los pacientes con EB. No se menciona en los reportes si se utilizó una cromoendoscopia digital en el momento de la sospecha de EB; tampoco hay descripciones del patrón mucoso. La prevalencia endoscópica obtenida del EB fue del 0,73\% (22 casos de 3000).

\section{DISCUSIÓN}

Con respecto a países con características similares a las nuestras (países de Latinoamérica), es evidente la poca correlación endoscópico-patológica: mientras que en el presente estudio fue del 28,5\% en otros ha sido hasta del $40 \%$ (15); en el estudio realizado en México dicha correlación fue del $60 \%$ (11),y en el trabajo desarrollado en Perú se encontró una correlación muy similar del $25 \%$ ( 11 de 44 pacientes) (16).

Todo ello podría sugerir que las características endoscópicas del EB no son muy predictivas para el diagnóstico, que estas son muy variables (que se tratan del EB de segmento corto o incluso focal, lo cual dificulta una toma de biopsia precisa) o bien que no se ha tomado un número adecuado de biopsias en relación con el tamaño del posible EB observado (dato que no podemos conocer con certeza, puesto que en el $63 \%$ no se mencionó la longitud del segmento observado).

Aunque la mayoría de pacientes diagnosticados con EB tienen más de 60 años, el rango de edad en el cual se encuentran es amplio (de 40 a 79 años) y la desviación estándar es de 8 años. A partir de esto podemos deducir que no debe descartarse la patología solamente en mayores de 60 años, sino que se requiere visualizar con detenimiento el esófago en pacientes de cualquier edad. Además, en caso de ser necesario, se podría realizar la implementación de controles endoscópicos a pacientes con reflujo gastroesofágico desde una edad menor. La edad media obtenida concuerda con lo reportado en la literatura (17).

Por otro lado, resultó interesante que la relación entre hombres y mujeres se invirtiera. Contrario a lo que sugiere la literatura (17), la prevalencia fue mayor en el sexo femenino que en el masculino. En efecto, por cada hombre diagnosticado con EB, se confirmó la existencia de la patología en 2 mujeres. Este es un resultado inesperado, al cual le debemos prestar más atención a la hora de efectuar la evaluación general de los pacientes, ya que podría deberse a que, dentro de la población objeto, la mayoría eran mujeres (54\%).

Sin embargo, esta explicación pierde validez al tomar específicamente la población de cada institución. En la primera, 
la mayoría de pacientes diagnosticados fueron mujeres a pesar de que la población general estaba conformada por más hombres, y viceversa en la segunda institución. Queda entonces la duda sobre por qué se invirtió la relación.

Como sabemos, esta es una patología asociada, en gran medida, con el reflujo gastroesofágico crónico $(1,18)$. Los resultados obtenidos lo ratifican, dado que esta enfermedad digestiva fue la principal indicación para realizar una endoscopia en los pacientes en quienes se encontró el EB. No obstante, no podemos ignorar que en el otro $50 \%$ de los casos con EB se practicó una endoscopia por un motivo no relacionado con el reflujo gastroesofágico crónico. Esto podría sugerir que, en muchos casos, la patología no se manifiesta mediante síntomas específicos y que, independientemente de cuál sea la indicación de la endoscopia, se debe efectuar una visualización detallada del esófago en todos los casos.

Dentro de los datos obtenidos, la mayoría de los pacientes con EB tenían un IMC $>25 \mathrm{~kg} / \mathrm{m}^{2}$, lo que es concordante con la evidencia disponible, que manifiesta que existe un riesgo de 2 a 3 veces mayor de desarrollar la enfermedad si el paciente está en sobrepeso o con algún grado de obesidad $(19,20)$.

Entre tanto, el $50 \%$ de los pacientes con EB tuvieron antecedente de tabaquismo (ya sea como hábito abandonado o actual). Este hallazgo se relaciona con lo referido en la literatura, en la cual el tabaquismo (actual o previo) es un factor de riesgo para el desarrollo de la patología en mención $(21,22)$. En contraste, nuestro estudio no sugiere que el consumo de alcohol sea un factor de riesgo para EB, dado que la mayoría de los pacientes con diagnóstico no ingerían dichas bebidas, lo cual es concordante con la evidencia que menciona que este no es un factor de riesgo consistente.

Nos gustaría señalar también la falta de homogeneidad de las descripciones de posibles EB en los reportes de endoscopia. En el mundo, se han descrito herramientas que ayudan a caracterizar la lesión de manera objetiva; entre ellas, se encuentran los criterios de Praga para el EB de segmento largo. Estos tienen implicaciones en el pronóstico y el riesgo de desarrollo de cáncer o en la clasificación por longitud del segmento observado (23). No obstante, más de la mitad de los reportes presentaban una vaga descripción de las lesiones, sin especificar qué longitud tenía el segmento. Incluso, algunos de ellos solo mencionaban el EB sin ninguna otra descripción detallada.

De modo contrario, en los estudios realizados en otros países, todos los casos confirmados tenían clasificación del EB (segmento largo y corto) $(11,16,24)$. Así, al considerar los 8 pacientes de nuestro estudio, en quienes sí se reportó la longitud, la cantidad de EB de segmento corto frente al largo fue la misma, mientras que en los estudios de otros países el EB de segmento corto fue 3 veces más frecuente que el largo $(16,24)$.

En consecuencia, es de gran importancia recalcar que estas herramientas deberían ser utilizadas como norma para describir las lesiones, dado que el uso de los criterios de Praga o la longitud de las lesiones permite darse una idea más amplia, a fin de evaluar el riesgo de adenocarcinoma y apoyar más sólidamente un posible tratamiento (23).

Así bien, en comparación con países con características similares a las nuestras (países de Latinoamérica), encontramos que la prevalencia en Colombia es muy baja, incluso menor de lo que esperábamos: tan solo del 0,73\%. La prevalencia en estudios similares realizados en México y en Chile fue más alta a la estimada en nuestro estudio: 1,8 (11) y $1,6 \%$ (24) respectivamente.

Sin embargo, en un estudio desarrollado en Perú, se estimó una prevalencia del 0,48 \% (16), cuyo valor es similar al encontrado en nuestro trabajo, con una muestra de población también parecida. En ese sentido, la baja prevalencia documentada puede ser dada por las siguientes explicaciones posibles. En primer lugar, es probable que, realmente, la prevalencia del EB en la población colombiana sea tan baja, cuya cifra se asemeja a la de Japón, que alcanza el 0,9-1,2 \% (25). De hecho, en este país asiático algunos estudios han propuesto como explicación a dichos valores una alta prevalencia de infección por Helicobacter pylori (25), lo cual también podría ser aplicable en nuestro contexto. Además, como ya lo referimos, la prevalencia de nuestro estudio es similar a la reportada en Perú.

En segunda instancia, podría suceder que el tiempo abarcado con la toma de datos sea muy corto: los 1500 datos de una de las instituciones correspondían únicamente a 20 días. En vista de que el EB es una patología con una prevalencia tan baja, es posible que, en ese corto período, se presentaran aleatoriamente muy pocos pacientes con la enfermedad.

Como tercera explicación, a pesar de que se utilizó un endoscopio de alta definición y de que los endoscopistas que realizaron el procedimiento en el presente estudio tienen un excelente desempeño en dicho campo, puede que el bajo diagnóstico endoscópico sea consecuencia de que no se proporcionó la suficiente importancia a la observación del esófago de pacientes en quienes la búsqueda no se enfocó primordialmente hacia el EB. Esto iría de la mano con la falta de uso de herramientas para la descripción de los hallazgos sugestivos de EB en la endoscopia.

Como cuarta explicación, sobre la base de la información escasa proporcionada en la mayoría de los reportes de endoscopia, no se logró determinar si se tomó el número adecuado de biopsias en relación con el tamaño del posible EB observado. Esto, igualmente, podría contribuir a falsos negativos en las biopsias y a una menor prevalencia que la real.

Dentro de las limitaciones del estudio, se encontró que, aunque se hizo una revisión cuidadosa y extensa, la población no fue completamente representativa, y que la elección de los lugares evaluados se dio a partir del criterio individual de los investigadores (posible sesgo de selección). Asimismo, 
pudo haber un riesgo de sesgo de memoria al indagar acerca de la magnitud exacta de una exposición en etapas previas de la vida (en este caso tabaquismo y consumo de alcohol), y un sesgo de confusión con variables no contempladas que expliquen la verdadera razón de ciertas asociaciones (por ejemplo, que al considerar alguna variable se concluyera que el EB no es más prevalente en mujeres).

Las implicaciones en el área de investigación son amplias. Con este estudio apuntamos a dar un primer paso en el reconocimiento de esta entidad en la población colombiana, así como a conocer las posibles falencias relacionadas con el diagnóstico de la enfermedad. Ahora, con estos resultados, pretendemos realizar un segundo estudio en el cual se implementen herramientas de diagnóstico, como la longitud del segmento y los criterios de Praga, y se efectúe una visualización más detallada del esófago. Lo anterior, con el fin de observar si a partir de la implementación de estas herramientas resulta una mayor prevalencia y una correlación endoscópico-patológica, o si realmente la obtenida en este estudio es la prevalencia real.

\section{CONCLUSIONES}

En las instituciones tomadas, el EB es una patología de muy baja prevalencia, predominante en mujeres de edad avan- zada, con síntomas de reflujo gastroesofágico, sobrepeso u obesidad y antecedente de tabaquismo. En general, luego de la comparación de datos obtenidos por otros estudios, podemos concluir que, aunque la prevalencia en países como Chile y México es mayor a la obtenida en nuestro estudio, las características demográficas son similares, con la excepción del sexo $(11,16,24)$.

Es importante señalar que la muestra poblacional es mucho menos significativa en los estudios mencionados, en comparación con nuestra muestra. Los resultados descritos en Perú se asemejan a los nuestros tanto en la prevalencia como en las características demográficas y la correlación endoscópico-patológica (16).

\section{Agradecimientos}

Agradecemos a las dos instituciones en las cuales se realizó el estudio, en especial al servicio de gastroenterología de cada una.

\section{Fuente de financiación}

Ningún grupo, institución u organización financió este proyecto, el cual fue realizado, en su totalidad, por los autores. El estudio no requirió de ninguna financiación.

\section{REFERENCIAS}

1. Spechler SJ, Souza RF. Barrett's esophagus. N Engl J Med. 2014;371(9):836-845. http://doi.org/10.1056/NEJMra1314704

2. Hani A. Esófago de Barrett: Una problemática para analizar, editorial. Rev Col Gastroenterol. 2008;23(1):1-2.

3. Van der Veen AH, Dees J, Blankensteijn JD, Van Blankenstein M. Adenocarcinoma in Barrett's oesophagus: an overrated risk. Gut. 1989;30(1):14-18. http://doi.org/10.1136/gut.30.1.14

4. Hvid-Jensen F, Pedersen L, Drewes AM, Sørensen HT, Funch-Jensen P. Incidence of adenocarcinoma among patients with Barrett's esophagus. N Engl J Med. 2011;365(15):1375-1383. http://doi.org/10.1056/NEJMoa1103042

5. Pohl H, Pech O, Arash H, Stolte M, Manner H, May A, Kraywinkel K, Sonnenberg A, Ell C. Length of Barrett's oesophagus and cancer risk: implications from a large sample of patients with early oesophageal adenocarcinoma. Gut. 2016;65(2):196-201. http://doi.org/10.1136/gutjnl-2015-309220

6. Sikkema M, Looman CW, Steyerberg EW, Kerkhof M, Kastelein F, van Dekken H, van Vuuren AJ, Bode WA, van der Valk H, Ouwendijk RJ, Giard R, Lesterhuis W, Heinhuis R, Klinkenberg EC, Meijer GA, ter Borg F, Arends JW, Kolkman JJ, van Baarlen J, de Vries RA, Mulder AH, van Tilburg AJ, Offerhaus GJ, ten Kate FJ, Kusters JG, Kuipers EJ, Siersema PD. Predictors for neoplastic progression in patients with Barrett's Esophagus: a prospective cohort study. Am J Gastroenterol. 2011;106(7):1231-8. http://doi.org/10.1038/ajg.2011.153

7. Yousef F, Cardwell C, Cantwell MM, Galway K, Johnston BT, Murray L. The incidence of esophageal cancer and high-grade dysplasia in Barrett's esophagus: a systematic review and meta-analysis. Am J Epidemiol. 2008;168(3):237-249. http://doi.org/10.1093/aje/kwn121

8. Sierra F. Incidencia de adenocarcinoma en esófago de Barrett, Fundación Santa Fe de Bogotá, 11 años de seguimiento. Rev Col Gastroenterol. 2008;23(1):13-25.

9. Desai TK, Krishnan K, Samala N, Singh J, Cluley J, Perla $S$, Howden CW. The incidence of oesophageal adenocarcinoma in non-dysplastic Barrett's oesophagus: a metaanalysis. Gut. 2012;61(7):970-6. http://doi.org/10.1136/gutjnl-2011-300730 
10. Bhat S, Coleman HG, Yousef F, Johnston BT, McManus DT, Gavin AT, Murray LJ. Risk of malignant progression in Barrett's esophagus patients: results from a large populationbased study. J Natl Cancer Inst. 2011;103(13):1049-57. http://doi.org/10.1093/jnci/djr203

11. Herrera JL, Monreal R, García D, González EI, Borjas OD, Maldonado HJ, González JA. Prevalencia de esófago de Barrett: estudio observacional en una clínica de gastroenterología. 2017;82(4):296-300. https://doi.org/10.1016/j.rgmx.2017.01.006

12. Olmos JA, Piskorz MM, Vela MF. Revisión sobre enfermedad por reflujo gastroesofágico (ERGE). Acta Gastroenterol Latinoam. 2016;46(2):160-172.

13. Gerson LB, Shetler K, Triadafilopoulos G. Prevalence of Barrett's esophagus in asymptomatic individuals. Gastroenterology. 2002;123(2):461-467. http://doi.org/10.1053/gast.2002.34748

14. Brunicardi FC, Andersen DK, Billiar TR, Dunn DL, Hunter JG, Matthews JB, Pollock RE. Schwartz's Principles of Surgery. Nueva York: McGraw-Hill, 9a edición; 2010.

15. Angarita OR, Granados CE, Ricaurte O. Frecuencia de esófago de Barrett en una serie de biopsias endoscópicas de esófago (2002-2007). Rev Col Gastroenterol. 2008;23(1):4-12.

16. Chacaltana A, Urday C, Ramon W, Rodríguez C, Espinoza J, Velarde H, Rodríguez I, Lucho E, Rauch E. Prevalencia, características clínico-endoscópicas y factores predictivos de Esófago de Barrett. Rev Gastroenterol Perú. 2009;29(1):24-32.

17. Odze RD. Gastrointestinal Pathology, An Issue of Gastroenterology Clinics (The Clinics: Internal Medicine). Filadelfia: Saunders; 2007.

18. Spechler SJ. Barrett esophagus and risk of esophageal cancer: a clinical review. JAMA. 2013;310(6):627-636. http://doi.org/10.1001/jama.2013.226450
19. Shinkai H, Iijima K, Koike T, Abe Y, Dairaku N, Inomata Y, Kayaba S, Ishiyama F, Oikawa T, Ohyauchi M, Ito H, Asonuma S, Hoshi T, Kato K, Ohara S, Shimosegawa T. Association between the body mass index and the risk of Barrett's esophagus in Japan. Digestion. 2014;90(1):1-9. http://doi.org/10.1159/000357776

20. El-Serag HB, Kvapil P, Hacken-Bitar J, Kramer JR. Abdominal obesity and the risk of Barrett's esophagus. Am J Gastroenterol. 2005;100(10):2151-2156. http://doi.org/10.1111/j.1572-0241.2005.00251.x

21. Iyer PG, Kaul V. Barrett Esophagus. Mayo Clin Proc. 2019;94(9):1888-1901. http://doi.org/10.1016/j.mayocp.2019.01.032

22. Vargas G. Prevalencia y factores de riesgo en el Hospital Nacional Arzobispo Loayza, Lima-Perú. Rev Gastroenterol. 2010;30(4):284-304.

23. Sharma P, Dent J, Armstrong D, Bergman JJ, Gossner L, Hoshihara Y, Jankowski JA, Junghard O, Lundell L, Tytgat GN, Vieth $M$. The development and validation of an endoscopic grading system for Barrett's esophagus: the Prague C \& M criteria. Gastroenterology. 2006;131(5):1392-9. http://doi.org/10.1053/j.gastro.2006.08.032

24. Csendes A, Smok G, Burdiles P, Quesada F, Huertas C, Rojas J, Korn O. Prevalence of Barrett's esophagus by endoscopy and histologic studies: a prospective evaluation of 306 control subjects and 376 patients with symptoms of gastroesophageal reflux. Dis Esophagus. 2000;13(1):5-11. http://doi.org/10.1046/j.1442-2050.2000.00065.x

25. Hongo M. Review article: Barrett's oesophagus and carcinoma in Japan. Aliment Pharmacol Ther. 2004;20 Suppl $8: 50-54$ http://doi.org/10.1111/j.1365-2036.2004.02230.x 